\title{
Erratum to: Computing translational diffusion and sedimentation coefficients: an evaluation of experimental data and programs
}

\author{
Mattia Rocco $^{1}$ (i) Olwyn Byron ${ }^{2}$
}

Published online: 18 July 2015

(C) European Biophysical Societies’ Association 2015

\section{Erratum to: Eur Biophys J \\ DOI 10.1007/s00249-015-1042-9}

In the original publication of the article, the minus sign is omitted in the last two entries of the last column "BEST Heur" in Table 2. Similar error is also found in Table 3, in the fifth entry under Column 7 . The correct version of both Tables 2 and 3 are given for your reading:

The online version of the original article can be found under doi:10.1007/s00249-015-1042-9.

Mattia Rocco

mattia.rocco@hsanmartino.it

1 Biopolimeri e Proteomica, IRCCS AOU San Martino-IST,

Istituto Nazionale per la Ricerca sul Cancro,

Largo R. Benzi 10, 16132 Genova, Italy

2 School of Life Sciences, College of Medical,

Veterinary and Life Sciences, University of Glasgow,

Glasgow G12 8QQ, UK 


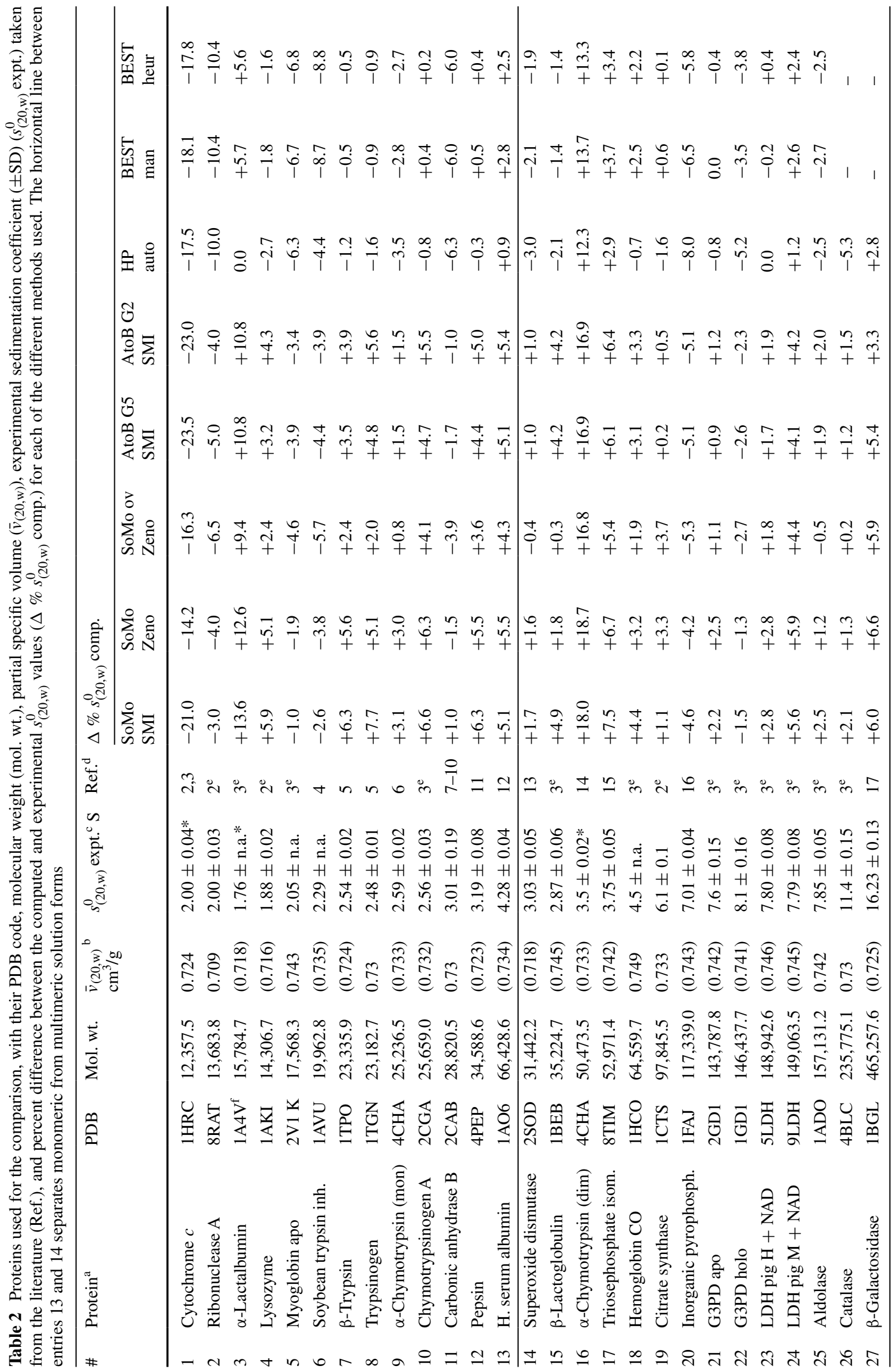




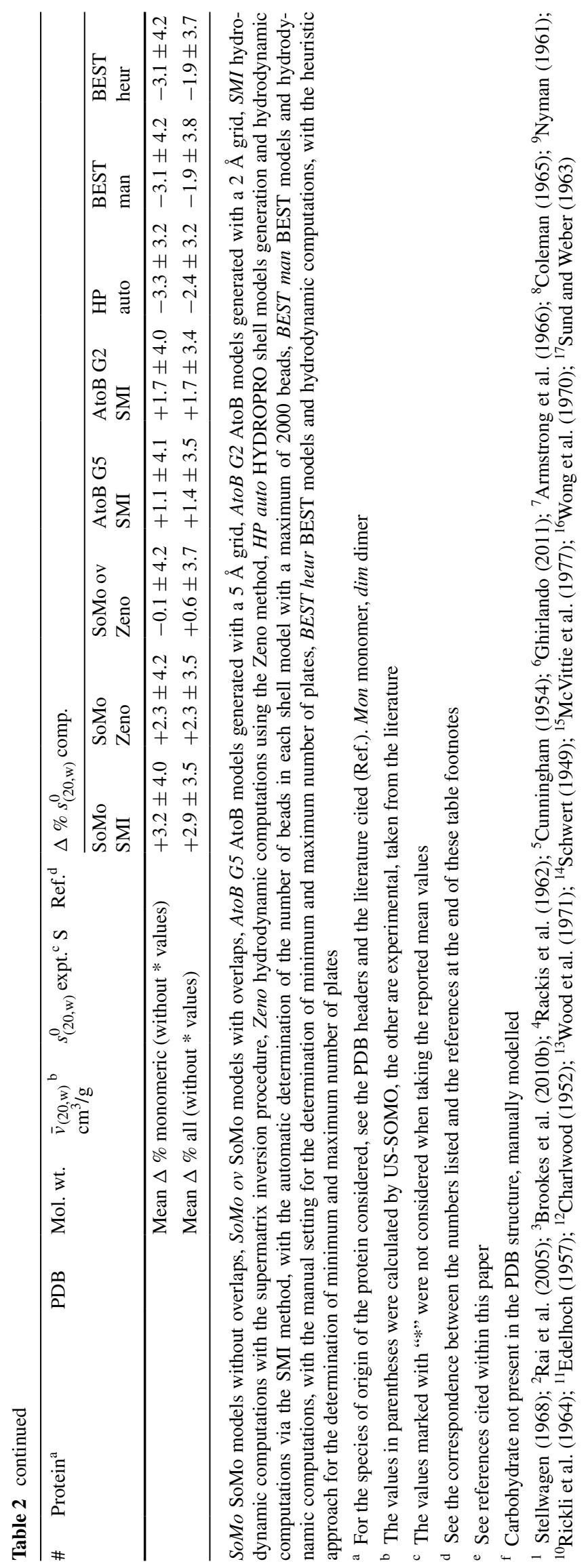


Table 3 Characteristics and performances of the main hydrodynamic modelling/computational methods discussed in this work

\begin{tabular}{|c|c|c|c|c|c|c|c|c|c|}
\hline \multirow[t]{2}{*}{ Program (conditions) } & \multirow[t]{2}{*}{$\begin{array}{l}\text { Structure } \\
\text { check? }\end{array}$} & \multirow[t]{2}{*}{$\mathrm{NMR}^{\mathrm{a}}$} & \multirow[t]{2}{*}{ Modelling method } & \multirow[t]{2}{*}{$\begin{array}{l}\text { Computational } \\
\text { method }\end{array}$} & \multirow{2}{*}{$\begin{array}{l}D_{t(20, \mathrm{w})}^{0} \\
\text { average } \\
\Delta \%^{\mathrm{b}}\end{array}$} & \multirow{2}{*}{$\begin{array}{l}s_{(20, \mathrm{w})}^{0} \\
\text { average } \\
\Delta \%^{\mathrm{c}}\end{array}$} & \multicolumn{3}{|c|}{$\begin{array}{l}\text { Computing time (minutes) for } \\
\text { selected structures }^{\mathrm{d}}\end{array}$} \\
\hline & & & & & & & $\begin{array}{l}1 \mathrm{AKI} \\
(14 \mathrm{kDa})\end{array}$ & $\begin{array}{l}1 \mathrm{AO} 6 \\
(66 \mathrm{kDa})\end{array}$ & $\begin{array}{l}1 \mathrm{ADO} \\
(160 \mathrm{kDa})\end{array}$ \\
\hline $\begin{array}{l}\text { SoMo (with overlaps) } \\
\text { in US-SOMO }\end{array}$ & $\mathrm{Yes}^{\mathrm{e}}$ & Yes & $\mathrm{BM}$, residue to bead & Zeno & $-0.2 \pm 2.4$ & $-0.6 \pm 3.7$ & 0.5 & 7.8 & 20.3 \\
\hline $\begin{array}{l}\text { AtoB ( } 5 \AA \text { grid) in } \\
\text { US-SOMO }\end{array}$ & $\mathrm{Yes}^{\mathrm{e}}$ & Yes & $\mathrm{BM}$, grid & SMI & $+0.3 \pm 2.4$ & $+1.4 \pm 3.5$ & 0.03 & 1 & 9 \\
\hline $\begin{array}{l}\text { SoMo (no overlaps) } \\
\text { in US-SOMO }\end{array}$ & Yes $^{\mathrm{e}}$ & Yes & $\mathrm{BM}$, residue to bead & SMI & $+1.9 \pm 2.5$ & $+2.9 \pm 3.5$ & 0.02 & 0.2 & 0.5 \\
\hline $\begin{array}{l}\text { BEST (manual) in } \\
\text { US-SOMO }\end{array}$ & Yes $^{f}$ & No & $\begin{array}{l}\text { BE }(2000-6000 \\
\text { plates })\end{array}$ & SI & $-2.7 \pm 2.1$ & $-1.9 \pm 3.8$ & 356 & 100 & 93 \\
\hline $\begin{array}{l}\text { BEST (heuristic) in } \\
\text { US-SOMO }\end{array}$ & Yes $^{f}$ & No & $\begin{array}{l}\mathrm{BE} \text { (variable \# of } \\
\text { plates) }\end{array}$ & SI & $-2.8 \pm 2.2$ & $-1.9 \pm 3.7$ & 170 & 261 & 1072 \\
\hline $\begin{array}{l}\text { HYDROPRO } \\
\text { (WinHydropro) }\end{array}$ & No & No & $\begin{array}{l}\text { Shell BM }(\leq 2000 \\
\text { beads })\end{array}$ & SMI & $-3.6 \pm 3.0$ & $-2.4 \pm 3.2$ & 0.3 & 0.3 & 0.3 \\
\hline
\end{tabular}

$B M$ bead modelling, $B E$ boundary elements, $S M I$ supermatrix inversion, $S I$ surface integrals

a Automatic computation and averaging of hydrodynamic parameters possible for multiple structures in NMR-type files

${ }^{\mathrm{b}}$ For all test proteins listed in Table 1, outliers excluded $( \pm \mathrm{SD})$

${ }^{c}$ For all test proteins listed in Table 2, outliers excluded $( \pm \mathrm{SD})$

${ }^{\mathrm{d}}$ For the Zeno and SMI methods within US-SOMO (Windows version) and HYDROPRO (WinHydropro), computations were run on an Intel Core i5-3470 3.2 GHz PC with 6 GB RAM, operating under the Windows 7 Professional OS; for BEST within US-SOMO, they were run on the TACC Stampede cluster, and do not include waiting times in the queue (see "Materials and methods")

e Approximate methods available for non-coded or incomplete residues

${ }^{\mathrm{f}}$ Checks performed but no influence on program execution 